\title{
B-CELL FAILURE AND PRESERVATION IN TYPE 2 DIABETES MELLITUS: A REVIEW
}

\author{
Ksh. Achouba Singh'1, R. K. Banashree Devi², Kh. Lokeshwar Singh ${ }^{3}$
}

${ }^{1}$ Associate Professor, Department of Endocrinology, Jawaharlal Nehru Institute of Medical Sciences (JNIMS), Porompat, Imphal. ${ }^{2}$ Associate Professor, Department of Pathology, Jawaharlal Nehru Institute of Medical Sciences (JNIMS), Porompat, Imphal. ${ }_{3}^{3}$ Associate Professor, Department of Medicine, Jawaharlal Nehru Institute of Medical Sciences (JNIMS), Porompat, Imphal.

\section{ABSTRACT}

Progressive decline in $\beta$-cell function and $\beta$-cell mass is associated with prolonged exposure to insulin resistance environment in patients of T2DM, more prominently in obese diabetics at the level of skeletal muscle, adipose tissue and liver. Here, a review of the available evidences of therapeutic regimes to preserve and maintain the lifespan of $\beta$-cells of pancreas has been made.

\section{KEYWORDS}

$\beta$ - Cell, Insulin Resistance, Type 2 Diabetes Mellitus, T2DM.

HOW TO CITE THIS ARTICLE: Singh KA, Devi RKB, Singh KL. B-Cell failure and preservation in type 2 diabetes mellitus: a review. J. Evolution Med. Dent. Sci. 2016;5(72):5315-5321, DOI: 10.14260/jemds/2016/1204

\section{BACKGROUND}

DM is now considered one of the most common noncommunicable diseases in the world causing $5 \%$ of all deaths per year. Regardless of its aetiology, the end point of DM is $\beta$ cell death. Therefore, new therapies should aim at preventing $\beta$-cell death and promote $\beta$-cell regeneration in patients in order to improve blood glucose without treatment-derived side effects. Deterioration in $\beta$-cell function and mass in type 2 diabetics is progressive. Only about $50 \%$ of normal islet function remains at the time of diagnosis and a reduction in $\beta$-cell mass of about $60 \%$ was shown at necropsy. Accelerated apoptosis is considered as the cause of the reduction of $\beta$-cell mass. The major factors for progressive loss of $\beta$-cell function and mass are glucotoxicity, lipotoxicity, overstimulation, proinflammatory cytokines, leptin and islet cell amyloid deposition. Impaired $\beta$-cell function and possibly reduced $\beta$ cell mass appear to be reversible, particularly at early stages of the disease. Insulin resistance and $\beta$-cell dysfunction both play important roles in the development and progression of T2DM.(1) It is evident that while insulin resistance lays the foundation for glucose intolerance, the progression to T2DM does occur in parallel to a degree of $\beta$-cell dysfunction, allowing Blood Glucose (BG) levels to rise.(2) Analysis of the results of the UKPDS and those of other studies suggests that pertaining to the diagnosis of T2DM, decreasing $\beta$-cell function is almost entirely responsible for progression of the disease.(3) At time of entry into the UKPDS, those who were newly diagnosed with diabetes had a reduction in insulin sensitivity of approximately $40 \%$ and a reduction in $\beta$-cell function of approximately 50\%. Over the course of the study, however, $\beta$-cell function continued to decline as calculated by Homeostasis Model Assessment (HOMA), while insulin sensitivity remained relatively constant. Pharmacological interventions utilised in the UKPDS were largely unsuccessful

Financial or Other, Competing Interest: None.

Submission 17-08-2016, Peer Review 29-08-2016,

Acceptance 01-09-2016, Published 08-09-2016.

Corresponding Author:

Dr. Ksh. Achouba Singh,

Uripok Bachaspati Maning Leikai,

Imphal-795001, Manipur, India.

E-mail: drachoubasingh@yahoo.com

DOI: $10.14260 /$ jemds/2016/1204 in maintaining BG control, while on follow-up that is associated with progression of type 2 diabetes.(4) Subsequent to 3 years of monotherapy, approximately $50 \%$ of the treatment group attained the study goal of $\mathrm{HbA} 1 \mathrm{c}<7.0 \%$, whereas 9 years later, only $25 \%$ achieved this goal with monotherapy.(6) The majority of patients required combined antidiabetic agents to achieve their BG targets.

The challenges associated with BG control in the management of T2DM have far reaching importance for longterm outcomes associated with the disease. In the UKPDS, a mere $0.9 \%$ reduction of $\mathrm{HbA} 1 \mathrm{c}$ produced a statistically significant $25 \%$ decrease in the incidence of microvascular complications. ${ }^{(5)}$

A growing body of evidence suggests that macrovascular complications may be more closely related to insulin resistance status.(6) It would therefore appear that an ideal pharmacological agent for the prevention of the long-term complications of type 2 diabetes would be to preserve $\beta$-cell function and reduce levels of insulin resistance. Of these characteristics, the capacity of pharmacological agents to preserve $\beta$-cell function is not well discovered. It has been suggested that the ability to prevent or at least retard the rise of BG with increased duration of diabetes may be facilitated by newly approved antidiabetic medications that were not available in the UKPDS. Interestingly, reducing insulin demand is expected to retard the progression from the insulin resistant stage to Impaired Glucose Tolerance (IGT) and diabetes.

\section{Deterioration of B-Cell}

In order to identify pharmacological agents that may have therapeutic potential for the prevention or slowing of the progression of type 2 diabetes. Many hypotheses have attempted to explain the aetiology of declining $\beta$-cell function from glucotoxicity and/or lipotoxicity to overstimulation of $\beta$-cells and amyloid deposition. Relation between declining insulin production and functional decline pertaining to cellular mass or diminished $\beta$-cell function has also been investigated.(7) In addition to providing an understanding of the physiological mechanisms that lead to $\beta$-cell dysfunction, the unique genetic inheritance of individuals who are prone to develop type 2 diabetes as a result of susceptibility to these factors should also be considered. 


\section{Glucotoxicity}

Temporary lack of insulin response during periods of hyperglycaemia referred to as glucose desensitisation have been demonstrated and results from a transient impairment of insulin secretion with associated hyperglycaemia and responds favourably to pharmacological treatment with typical antihyperglycaemic agents.(8) Alternatively, glucose toxicity refers to an irreversible compromise in insulin synthesis resulting from prolonged elevation of BG. Adaptation to chronic hyperglycaemia occurs through increased $\beta$-cell mass enhancing the increase in insulin secretion.(9) The results of studies have suggested that glucotoxicity nullifying the adaption to chronic hyperglycaemia, results from chronic oxidative stress adversely affecting insulin gene transcription and promoting accumulation of AGEs, which also affects transcription of the insulin gene and leads to $\beta$-cell destruction.(10)

\section{Overstimulation}

B-cells are chronically stimulated by chronic hyperglycaemia leading to overstimulation, which has been linked in experimental models to abnormally increase in cytoplasmic $\mathrm{Ca} 2+$.(11) This may trigger apoptosis of $\beta$-cells and contribute to irreversible loss of overall $\beta$-cell function. Since insulin resistance and the resultant hyperinsulinaemia occur long before the onset of type 2 diabetes, this hypothesis suggests that the process of $\beta$-cell destruction occurs well before a diagnosis of diabetes in genetically susceptible individuals.(12) This theory is supported by the observation that when $\beta$-cells are rested, pancreatic endogenous insulin secretion is improved on administration of exogenous insulin.(13) At the same time, amelioration of insulin resistance will give rest to B-cells by reducing the requirement for insulin secretion.

\section{Lipotoxicity}

In persistent high levels fatty acids increase basal insulin levels, but inhibit glucose-induced insulin secretion. The results of studies also suggest that excess fatty acids cause $\beta$ cell death through apoptosis and along with raised BG levels; insulin gene expression is inhibited. Hypotheses arising from these studies suggest that coexisting elevated fatty acid levels and hyperglycaemia prevent normal oxidation of fatty acids in the mitochondria and esterification metabolites inhibit glucose-induced insulin secretion and insulin gene expression. Furthermore, type 2 diabetes results in a very high risk of dyslipidaemia in concert with the insulin resistance syndrome. Considered together, uncontrolled BG and lipid levels would promote progressive $\beta$-cell dysfunction in people with T2DM. More studies are on the way to evaluate the contribution of fatty acids to the deterioration of $\beta$-cell function. Since changes in $\beta$-cells are more consistently correlated with increased BG levels than with fatty acid levels, fatty acids are considered as contributor to progressive $\beta$-cell dysfunction while glucose toxicity plays a more consistent and aetiological role.

\section{Amyloid Deposits}

Amylin is normally produced and secreted by the $\beta$-cell along with insulin.(14) As insulin levels rise in response to elevated insulin resistance, amylin levels rise in tandem resulting in hyperamylinaemia.(15) Amyloid deposits are present in the islet cells of up to $90 \%$ of individuals with type 2 diabetes.(16) Postmortem studies have revealed a 40 to $60 \%$ reduction in $\beta$-cell mass in individuals with type 2 diabetes.

Amyloid deposits are presumed to progressively replace $\beta$-cell, contributing to the progression of type 2 diabetes. Suggested mechanism is that elevated levels of amylin cause apoptosis of the $\beta$-cell, causing self-aggregation and polymerisation to the extracellular matrix within the islet. Based on research in mice, it has been suggested that high intake of dietary fat may play an important role in amyloid formation through alteration of islet $\beta$-cell polypeptide production. Study results suggest that amyloid deposition may precede the onset of declining $\beta$-cell function and type 2 diabetes. Increasing evidence points to the cytotoxicity of Islet Amyloid Polypeptide (IAPP) aggregates, which plays a major role in the loss of $\beta$-cell mass in type 2 diabetes. Thus, $\beta$-cell survival and function may be increased by preventing IAPP formation.

\section{Influence of Genetic Makeup on $\boldsymbol{\beta}$-Cell Failure}

The progression from a state of insulin resistance to a diagnosis of type 2 diabetes is likely dependent on interplay of environmental and genetic factors. Many genome studies support the hypothesis that susceptibility genes exist and promote $\beta$-cell dysfunction, thereby increasing the risk of type 2 diabetes.(17) Population studies have demonstrated that first-degree relatives of individuals with type 2 diabetes are at a higher risk of developing type 2 diabetes. A study of 531 first degree relatives of people with diabetes who had never had history of impaired BG control revealed that only $45 \%$ had normal glucose tolerance.(18) The genetic selectivity of individuals with elevated insulin resistance to be susceptible to progressive deterioration of $\beta$-cell function is a focus of intriguing research that is still in its primary stages.

\section{Therapeutic Options: Potential for $\beta$-cell Preservation} In light of the aetiologies of $\beta$-cell dysfunction, it is important to consider the influence of different classes of antihyperglycaemic agents on the progression of type 2 diabetes.

\section{Lifestyle Modification}

Weight reduction and regular physical activity have been shown to increase insulin sensitivity.(19) The Diabetes Prevention Program (DPP) was conducted to assess whether lifestyle intervention (Targeting at least a 7\% reduction in weight and 150 minutes of physical activity per week) or metformin (850 mg BID) therapy would prevent or delay the onset of type 2 diabetes in people with IGT.(20) After an average follow-up of 2.8 years, lifestyle intervention reduced the incidence of diabetes by $58 \%$, while metformin therapy reduced the incidence of diabetes by $31 \%$ compared to placebo. In the UKPDS, metformin did not prevent progression of diabetes in people already diagnosed with type 2 diabetes, but delayed the process compared to diet alone. The DPP illustrates the importance of lifestyle intervention in $\beta$-cell preservation in all phases of the disease process.

\section{Metformin}

Metformin's primary mechanism of action is to decrease hepatic glucose production. Metformin also decreases total LDL-C, Free Fatty Acid (FFA) and Triglyceride (TG) levels and 
slightly increases peripheral insulin sensitivity.(21) Metformin does not cause a gain in body weight. After a period of only 3 years, $50 \%$ of the patients failed to achieve the HbA1c target in the UKPDS. Metformin was the only treatment studied that demonstrated significant improvement in macrovascular outcomes in patients in the UKPDS (Obese patients only(22)). As noted in the DPP, metformin reduced the risk of a diagnosis of type 2 diabetes in patients with IGT by $31 \%$ vs. placebo compared to a $58 \%$ risk reduction with lifestyle intervention. The results of the UKPDS suggest that the mechanism behind this outcome does not prevent the ultimate progression of type 2 diabetes once diagnosed.

\section{Sulfonylureas}

Glyburide, chlorpropamide and gliclazide reduce high BG levels by stimulating the production of insulin by $\beta$-cells. $\mathrm{HbA1c}$ targets were not met with the use of sulfonylureas in the UKPDS in $50 \%$ of the patients after a period of 3 years. This observation may be associated with a number of factors. For example, apoptosis of $\beta$-cells has been associated with sulfonylurea treatment.(23) Furthermore, sulfonylureas have been shown to increase postprandial amylin concentrations.(24) There appears to be the potential for overstimulation of the $\beta$-cells by sulfonylureas, as these agents promote insulin secretion through a glucose-like effect on the potassium adenosine triphosphate (K+-ATP) channel. Whether this action would actually accelerate $\beta$-cell dysfunction remains to be verified in studies directly comparing long-term outcomes on $\beta$-cell function of sulfonylureas with agents that do not stimulate the $\beta$-cell, such as insulin sensitizers. However, after 6 months exenatide and sitagliptin had no significant effect on functional $\beta$-cell mass as measured by $\beta$-cell secretory capacity, whereas glimepiride shown to enhance $\beta$ - and $\alpha$-cell secretion. Sulfonylurea in the form of glyburide as initial monotherapy in type 2 diabetes showed an unfavourable combined changes in $\beta$-cell function and insulin sensitivity over time from an Oral Glucose Tolerance Test (OGTT) over a 4-year period as compared with rosiglitazone in the ADOPT follow-up study.(25)

\section{Meglitinides}

Although, the mechanism of action of the meglitinides, repaglinide and nateglinide, is through stimulation of $\beta$-cells, earlier insulin release and a shorter half-life result in less overall insulin release (and $\beta$-cell stimulation) than with sulfonylureas.(26) Improved postprandial BG control with the meglitinides has not improved HbA1c levels, so these agents are viewed as alternatives to sulfonylureas for those who cannot tolerate sulfonylureas due to increased hypoglycaemic events or impaired renal function.(27)

\section{Alpha-Glycosidase Inhibitors}

The AGI acarbose has been shown to reduce postprandial insulin secretion and improve insulin sensitivity in patients with IGT.(28) The STOP-NIDDM randomised 714 and 715 patients with IGT to acarbose $100 \mathrm{mg}$ TID or placebo TID, respectively. After a mean follow-up of 3.2 years, $32 \%$ of acarbose-treated patients and $42 \%$ of placebo-treated patients were diagnosed as having diabetes $(B G=11.1$ mmol/L after 2-hour OGTT, representing a statistically significant $24 \%$ reduction in the relative risk of developing diabetes with acarbose. Reductions in BG level and $\mathrm{HbA1c}$ achieved with acarbose are lower than those observed with other anti-hyperglycaemic agents. In STOP-NIDDM, 31\% of patients taking acarbose discontinued treatment primarily due to diarrhea and flatulence compared to $19 \%$ of those taking placebo.(29)

\section{Thiazolidinediones}

The results of studies utilising the HOMA suggest that TZDs may play a role in sustaining or improving $\beta$-cell function.(30) Pioglitazone and rosiglitazone significantly increase insulin sensitivity at the level of adipose tissue, skeletal tissue and the liver, inducing increased glucose disposal and $\beta$-cell rest. TZDs improve insulin sensitivity by activating the PPARgamma. This activation alters the transcription of several genes involved in glucose and lipid metabolism and energy balance, which also includes those that code for lipoprotein lipase, fatty acid transporter protein, adipocyte fatty acid binding protein, fatty acyl-CoA synthase, malic enzyme, glucokinase and GLUT4.(31) This mechanism of action enables TZDs to cause significant reductions in FFAs and



improve lipid profiles through increases in HDL and production of larger, more buoyant LDL-C particles which are less atherogenic. Optimal environment for limiting the role of glucose toxicity in the evolution of $\beta$-cell dysfunction would be to maintain blood glucose level under control in a continuous manner. Treatment protocols in the UKPDS were not designed to control BG levels throughout the day; all therapeutic decisions were based on Fasting Blood Glucose (FBG) levels.(32) Although, the UKPDS demonstrated that lowering $\mathrm{HbA1c}$ by $0.9 \%$ significantly reduced the risk of microvascular complications and to consider is whether the outcomes may have been improved had BG control been maintained more consistently throughout the day.

In the DECODE study, use of the World Health Organisation's diagnostic criteria for diabetes included postprandial BG levels resulted in a much higher percentage of patients being diagnosed with type 2 diabetes compared with those diagnosed using the American Diabetes Association's diagnostic criteria (FBG alone). Furthermore, increased mortality was associated with increasing BG levels after a 2-hour postprandial in groups of patients with similar FBG levels, suggesting that FBG alone does not identify 
individuals at increased risk of complications associated with hyperglycaemia.(33) A study of 371 outpatients with type 2 diabetes who were treated with diet, metformin, a sulfonylurea or metformin and a sulfonylurea found that many patients with satisfactory HbA1c $(<7.0 \%)$ had high postprandial BG levels. The majority of patients had BG levels higher than normal and/or exaggerated postprandial BG excursions. Conclusion was that BG levels are the result of the ability of the pancreatic $\beta$-cells to respond to glucose stimulation and the ability of peripheral tissues to dispose of glucose post-prandially. Medications to directly modify postprandial BG levels include the meglitinides, acarbose and insulin. Greater reduction in HbA1c can be achieved by controlling BG levels throughout the day as illustrated in the Kumamoto Study. The ideal pharmacological management promoting long-term BG control would involve an agent or agents that target both FBG and non-fasting BG levels by allowing normal physiological secretion of insulin (i.e. restoration of $\beta$-cell function) while enhancing tissue insulin sensitivity. Metformin reduces hepatic glucose production with modest effect on peripheral insulin sensitivity of tissues and would therefore appear to have potential in preserving $\beta$ cell function. However, metformin did not attenuate the progression of type 2 diabetes in the UKPDS. Out of many antihyperglycaemic agents, only the TZDs have the potential to restore both insulin sensitivity and $\beta$-cell function in the long term. Although, it is well known that TZDs significantly improve tissue insulin sensitivity, less evidence of their effects on $\beta$-cell function is available.

\section{Effect of TZDs on B-Cell Response}

Evidence of the positive effects of TZDs therapy on $\beta$-cell function is available from a number of randomised, controlled trials utilising these agents as monotherapy or in combination with metformin or a sulfonylurea. A study comparing resting and exercise-stimulated skeletal muscle glucose uptake using euglycaemic-hyperinsulinaemic clamp measurement in patients with newly diagnosed type 2 diabetes revealed an increase in whole-body glucose uptake of $44 \%$ in rosiglitazone-treated patients at rest and a $99 \%$ increase during exercise-induced insulin stimulation compared to baseline ( $p<0.01$ and $p<0.0001$, respectively) Patients treated with metformin did not experience any changes in skeletal muscle or whole-body insulin sensitivity.(34) Monotherapy with pioglitazone and rosiglitazone in 23- to 26-week trials demonstrated significant 47 to $60 \%$ improvements in $\beta$-cell response compared to placebo,(35) 16-week trials evaluating TZDs as monotherapy or in addition to a sulfonylurea or metformin, significant reduction in insulin resistance occurred, but differences in $\beta$-cell response compared to placebo did not reach statistical significance.(36)

\section{Combination Therapy with TZDs: $\beta$-Cell Response}

Randomised, controlled trials have shown that improvements in $\beta$-cell response and insulin resistance occur when TZDs are added to either metformin or a sulfonylurea. The combination of a sulfonylurea and a TZD is logical, as sulfonylureas may hasten $\beta$-cell dysfunction over time, while TZDs may attenuate this effect. Furthermore, sulfonylureas may aid the BG-lowering effects of TZDs by increasing insulin levels.(37) Combination of a TZD and metformin is quite logical, except for the late stages of type 2 diabetes when insufficient pancreatic insulin production may preclude TZD performance. In such situation, insulin initiation would be needed. The activities of TZDs and metformin are complementary to each other in improving $\beta$-cell response. Mechanisms may include a better normalisation of BG levels (i.e. reduced glucose toxicity), fatty acid levels (i.e. reduced lipotoxicity) and insulin resistance (i.e. $\beta$-cell rest and reduced amylin production).(38) Combining a TZD with insulin also has merit. Addition of rosiglitazone $8 \mathrm{mg}$ daily demonstrated significantly reduced $\mathrm{HbA1c}$ from baseline by $1.2 \%$ ( $\mathrm{p}<0.0001$ ) after 26 weeks, despite a $12 \%$ mean reduction in insulin dose in a randomised, placebo-controlled trial in 319 patients with type 2 diabetes inadequately controlled with insulin monotherapy.(39)

\section{Improved $\beta$-Cell Function with TZDs}

Randomised, placebo-controlled trials demonstrated that in patients with type 2 diabetes, TZDs consistently improve $\beta$ cell response, reduce insulin resistance and improve HbA1c compared to placebo and/or baseline values when used alone or in combination or insulin. Other studies with troglitazone, a TZD not released in Canada and removed from the market in the United States due to concerns of hepatic toxicity have shown similar outcome.(40) The criteria for a pharmacological agent that could potentially preserve $\beta$-cell function are thus fulfilled. By extension, such an agent could play an important role in slowing or halting the progression of type 2 diabetes, which results from a continued decline in $\beta$-cell function. Long-term studies are required and many studies are currently underway to test the proposition that long-term control of BG is afforded by the TZDs as a result of $\beta$-cell preservation. ADOPT trial on type 2 diabetes followed for a 4-year period to compare the long-term effects of daily treatment with rosiglitazone $8 \mathrm{mg}$, metformin $2 \mathrm{~g}$ /day and glyburide $15 \mathrm{mg}$ using the primary outcome of monotherapy failure (Defined as FBG $>10 \mathrm{mmol} / \mathrm{L}$ ) has shown a significant improvement in favour of Rosiglitazone. Diabetes Reduction Approaches with Ramipril and Rosiglitazone Medications (DREAM) study has also shown a similar result of treatment Rosiglitazone or an angiotensin converting enzyme inhibitor in the prevention of progression to type 2 diabetes in patients with glucose intolerance. Unfortunately, studies examining the potential of acarbose for preserving $\beta$-cell function in patients with type 2 diabetes have not been conducted.

\section{Rational of Early Insulin use in B-Cell Preservation}

Insulin therapy allows for $\beta$-cell rest and subsequent improvement in $\beta$-cell function in type 2 diabetes when multiple injections are given for approximately 9 weeks. Further improvement in $\beta$-cell function is not realised if insulin is administered for longer periods of time. Reduction in basal amylin concentrations has been observed in type 2 diabetes treated with insulin as compared to diet therapy alone, but had no effect on postprandial amylin concentrations. While several strategies can be employed to tackle many of these factors contributing to $\beta$-cell decline, insulin alone has the most salutary effect on majority of them. Both acute and prolonged hyperglycaemia adversely affects $\beta$-cell function. Early use of insulin results in increased insulin gene expression and insulin synthesis. It provides rest to $\beta$-cells, already stretched to their capacity and helps them regenerate over time. B-cells are most stressed and therefore 
most vulnerable to programmed cell death (Apoptosis) during the first few months of clinical onset of diabetes. Rapid restoration of euglycaemia by early insulin therapy at this stage will naturally preserve $\beta$-cell function on a long-term basis. This has been demonstrated in several experimental and clinical studies. In Chinese hamster, a spontaneous and selectively inbred animal model for non-obese type 2 diabetics, rapid normalisation of glycaemia resulted in significant improvement in $\beta$-cell function. This was characterised by improved $\beta$-cell signalling induced by the cyclic AMP protein kinase A pathway. This was also associated with improved islet insulin content and $\beta$-cell morphology as demonstrated by immunocytochemistry. In patients with LADA, early initiation of insulin has been shown to preserve $\beta$-cell function as evidenced by C-peptide response higher in insulinised group than Sulfonylurea (SU) group, which showed significantly lesser C-peptide after two years. This worsened further at the end of three years. It has also been demonstrated that short-term glycaemic control by insulin infusion restores SU sensitivity in significant proportion of non-obese SU non-responsive type 2 diabetic subjects. During the 6-month follow-up period, they could be managed with glibenclamide alone. Metabolic improvement in the form of improved fasting and post-meal C-peptide levels as well. $\beta$-Cell function preservation can be achieved for at least 3.5 years with early intensive therapy for type 2 diabetes with either insulin, insulin plus metformin or triple oral therapy.

\section{Incretins and Incretin Mimetics}

GIP and GLP-1 are two incretin hormones produced by the $\mathrm{K}$ and L-cells of the intestine, respectively. These incretin secretion are glucose dependent and potentiate glucoseinduced insulin secretion from pancreatic $\beta$-cells in healthy individuals. However, both peptides are rapidly degraded by DPP-IV.(41) In view of their insulinotropic properties, major efforts have developed either mimetics of incretins, which are resistant to inhibitors of DPPIV. Historically, GIP was rapidly discarded as a therapeutic target due to its impaired insulinotropic effect in T2DM patients. Recent evidence suggests that GIP may be a promising target for the preservation and regeneration of $\beta$-cell mass in DM. Indeed, GIP was shown to be important for $\beta$-cell development as well as postnatal islet mass expansion and function. Transgenic mice expressing a dominant negative GIP receptor, specifically in pancreatic $\beta$-cells displayed an early disturbance in pancreatic islet development with a severe reduction in the $\beta$-cell mass with a commensurate increase in $\alpha-, \delta$ - and PP-cells.(42) Adult transgenic animals had reduced number of islets and $\beta$ cells as compared to control groups. Minimal postnatal islet expansion occurred due to a reduction in islet neogenesis. The changes in islet cell proliferation and apoptosis only marginally modified as compared to control mice. $\beta$-cell survival was shown to be increased in mouse islet with GIP in response to glucolipotoxicity through activation of the PI3K/PKB pathway and down-regulation of the pro-apoptotic factor Bax, stimulate expression of the anti-apoptotic Bcl-2.(43) In addition, GIP studies using human islets revealed that GIP could partially block cytokine-mediated cell death providing protective effects to this incretin. Interestingly, this antiapoptotic effect appeared to be conveyed by GIP-mediated increases in islet osteopontin.(44) Consistent with these in vitro data, administration of a DPP-IV-resistant analogue of GIP to rats blunted streptozotocin induced islet $\beta$-cell destruction and development of hyperglycaemia. Also observed the effect of GIP analogue to preserve $\beta$-cell mass in ZDF rats through decreased apoptosis.(45) In vitro, newborn rat islet $\beta$-cells proliferation was observed on stimulation with GIP, a mechanism potentially involving the activation of cyclin D1. Thus, GIP analogues resistant to DPP-4 cleavage may represent a promising new class of therapeutic agents which can enhance as well as preserve the critical $\beta$-cell mass required to maintain normoglycaemia in DM patients. LIBRA Trial, a double-blind, randomised, parallel-arm, placebocontrolled study assessed the capacity of liraglutide (GLP-1 agonists) to preserve b-cell function in patients with early T2DM who underwent 4 weeks of intensive insulin therapy before randomisation to either liraglutide or matching placebo and were followed for 48 weeks. It was found that Liraglutide enhanced $\beta$-cell function strongly that sustained over 48 weeks in early T2DM, but lost upon cessation of therapy.(46)

\section{Immune Therapy}

For the past 3 decades, a variety of immune interventions have been evaluated in newly detected T1D including nonspecific immunosuppression, pathway-specific immune modulation, antigen-specific therapies and cellular therapies. No single intervention has produced durable remission off therapy in most treated patients, but a valuable insights into disease mechanisms and potential immunologic correlation has been highlighted. T-cell-directed therapies including therapies that lead to partial depletion or modulation of effector and regulatory $\mathrm{T}$ cells have shown the most success and will likely form the backbone of future approaches. No immune interventions for preservation of $\beta$-cells of pancreas in T2D have so far been conducted.

\section{CONCLUSION}

Evidences are there to suggest the risk of macrovascular disease is closely tied to insulin resistance, while microvascular disease is associated with BG control. The evidence presented in this review suggests that preservation of $\beta$-cell function could slow or prevent progression of type 2 diabetes by maintaining a physiological response to elevated BG throughout the day. A single pharmacological agent that could preserve $\beta$-cell function and reduce insulin resistance would address mechanisms of both microvascular and macrovascular complications associated with T2DM. Such an agent if available would ideally be initiated in the early stages of type 2 diabetes or when glucose intolerance is evident, so that progression of the disease could be arrested before associated complications become a medical concern. To date, the longest published trial of TZD (Rosiglitazone) has reported maintenance of reductions in HbA1c and FFA levels throughout 30 months. Early initiation of insulin in the course of type 2 diabetes is highly rational based on available data. Incretins as a class of antidiabetic show, a promise in the preservation of $\beta$-cell function in T2DM.

\section{REFERENCES}

1. Kahn SE. The importance of the beta-cell in the pathogenesis of type 2 diabetes mellitus. Am J Med 2000;108(Suppl 6a):2S-8S. 
2. DeFronzo RA. Lilly lecture 1987 . The triumvirate: betacell, muscle, liver. A collusion responsible for NIDDM. Diabetes1988;37(6):667-87.

3. Gerich JE. Addressing the insulin secretion defect: a logical first-line approach. Metabolism 2000;49(10Suppl2):12-6.

4. Turner RC, Cull CA, Frighi V, et al. Glycemic control with diet, sulfonylurea, metformin, or insulin in patients with type 2 diabetes mellitus: progressive requirement for multiple therapies (UKPDS 49). JAMA 1999;281(21):2005-12.

5. American Diabetes Association. Implications of the United Kingdom prospective diabetes study. Diabetes Care 2002;25(Suppl 1):S28-S32.

6. Smiley T, Oh P, Shane LG. The relationship of insulin resistance measured by reliable indexes to coronary artery disease risk factors and outcomes-a systematic review. Can J Cardiol 2001;17(7):797-805.

7. Clark A, Jones LC, de Koning E, et al. Decreased insulin secretion in type 2 diabetes: a problem of cellular mass or function? Diabetes 2001;50(Suppl 1):S169-71.

8. Rosenblatt S, Miskin B, Glazer NB, et al. The impact of pioglitazone on glycemic control and atherogenicdyslipidemia in patients with type 2 diabetes mellitus. Coron Artery Dis 2001;12(5):413-23.

9. Weir GC, Laybutt DR, Kaneto H, et al. Beta-cell adaptation and decompensationduring the progression of diabetes. Diabetes 2001;50(Suppl 1):S154-9.

10. Poitout V, Robertson RP. Minireview: secondary beta-cell failure in type 2 diabetes-a convergence of glucotoxicity and lipotoxicity. Endocrinology 2002;143(2):339-42.

11. Grill V, Björklund A. Overstimulation and beta-cell function. Diabetes 2001;50(Suppl 1):S122-4.

12. Nyholm B, Porksen N, Juhl CB, et al. Assessment of insulin secretion in relatives of patients with type 2 (non-insulindependent) diabetes mellitus: evidence of early beta-cell dysfunction. Metabolism 2000;49(7):896-905.

13. Kärvestedt L, Andersson G, Efendic S, et al. A rapid increase in beta-cell function by multiple insulin injections in type 2 diabetic patients is not further enhanced by prolonging treatment. J Intern Med 2002;251(4):307-16.

14. Porte D, Kahn SE. Beta-cell dysfunction and failure in type 2 diabetes: potential mechanisms. Diabetes 2001;50(Suppl 1):S160-3.

15. Hayden MR, Tyagi SC. Remodeling of the endocrine pancreas: the central role of amylin and insulin resistance. South Med J 2000;93(1):24-8.

16. Bergman RN, Finegood DT, Kahn SE. The evolution of betacell dysfunction and insulin resistance in type 2 diabetes. Eur J Clin Invest 2002;32(Suppl 3):35-45.

17. Lindgren CM, Mahtani MM, Widén E, et al. Genomewide search for type 2 diabetes mellitus susceptibility loci in finnish families: the Botnia study. Am J Hum Genet 2002;70(2):509-16.

18. Jensen CC, Cnop M, Hull RL, et al. Beta-cell function is a major contributor to oral glucose tolerance in high-risk relatives of four ethnic groups in the U.S. Diabetes 2002;51(7):2170-8.
19. Pan XR, Li GW, Hu YH, et al. Effects of diet and exercise in preventing NIDDM in people with impaired glucose tolerance. The Da Qing IGT and diabetes study. Diabetes Care 1997;20(4):537-44.

20. Knowler WC, Barrett-Connor E, Fowler SE, et al. Reduction in the incidence of type 2 diabetes with lifestyle intervention or metformin. $\mathrm{N}$ Engl J Med 2002;346(6):393-403.

21. Rosenstock J. Improved insulin sensitivity and $\beta$-cell response suggested by HOMA analysis of pioglitazone therapy. Diabetologia 2000;43(Suppl 1):A1-316.

22. UK prospective diabetes study group. Effect of intensive blood-glucose control with metformin on complications in overweight patients with type 2 diabetes (UKPDS 34). Lancet 1998;352(9131):854-65.

23. Mandrup-Poulsen T. ß-cell apoptosis: stimuli and signaling. Diabetes 2001;50(Suppl 1):S58-S63.

24. Rachman J, Payne MJ, Levy JC, et al. Changes in amylin and amylin-like peptide concentrations and $ß$-cell function in response to sulfonylurea or insulin therapy in NIDDM. Diabetes Care 1998;21(5):810-6.

25 . Effects of rosiglitazone, glyburide, and metformin on $\beta$ cell function and insulin sensitivity in ADOPT. Diabetes 2011;60(5):1552-60.

26. Hollander PA, Schwartz SL, Gatlin MR, et al. Importance of early insulin secretion: comparison of nateglinide and glyburide in previously diet-treated patients with type 2 diabetes. Diabetes Care 2001;24(6):983-8.

27. Hanna AK, Woo V. Canadian diabetes association position paper on new oral hypoglycemic agents. Can J Diabetes Care 2001;25:152-6.

28. Chiasson JL, Josse RG, Leiter LA, et al. The effect of acarbose on insulin sensitivity in subjects with impaired glucose tolerance. Diabetes Care 1996;19(11):1190-3.

29. The STOP-NIDDM trial research group, Chiasson JL, Josse RG, et al. Acarbose for prevention of type 2 diabetes mellitus: the STOP-NIDDM randomised trial. Lancet 2002;359(9323):2072-7.

30. Reasner CA. Where thiazolidinediones will fit. Diabetes Metab Res Rev 2002;18(Suppl 2):S30-5.

31. Hauner $\mathrm{H}$. The mode of action of thiazolidinediones. Diabetes Metab Res Rev 2002;18(Suppl 2):S10-5.

32. Bonora E, Calcaterra F, Lombardi S, et al. Plasma glucose levels throughout the day and HbA1c interrelationships in type 2 diabetes: implications for treatment and monitoring of metabolic control. Diabetes Care 2001;24(12):2023-9.

33. The decode study group, on behalf of the european diabetes epidemiology group. Glucose tolerance and mortality: comparison of WHO and american diabetes association diagnostic criteria. Lancet 1999;354(9179):617-21.

34. Hällsten K, Virtanen KA, Lönnqvist F, et al. Rosiglitazone but not metformin enhances insulin and exercisestimulated skeletal muscle glucose uptake in patients with newly diagnosed type 2 diabetes. Diabetes 2002;51(12):3479-485.

35. Lebovitz HE, Dole JF, Patwardhan R, et al. Rosiglitazone monotherapy is effective in patients with type 2 diabetes. J Clin Endocrinol Metab 2001;86(1):280-8. 
36. Einhorn D, Rendell M, Rosenzweig J, et al. Pioglitazone hydrochloride in combination with metformin in the treatment of type 2 diabetes mellitus: a randomized, placebo controlled study. Clin Ther 2000;22(12):1395409.

37. Wolffenbuttel BH, Gomis R, Squatrito S, et al. Addition of low-dose rosiglitazone to sulphonylurea therapy improves glycaemic control in type 2 diabetic patients. Diabet Med 2000;17(1):40-7.

38. Fonseca V, Rosenstock J, Patwardhan R, et al. Effect of metformin and rosiglitazone combination therapy in patients with type 2 diabetes mellitus: a randomized controlled trial. JAMA 2000;283(13):1695-702.

39. Raskin P, Rendell M, Riddle MC, et al. A randomized trial of rosiglitazone therapy in patients with inadequately controlled insulin-treated type 2 diabetes. Diabetes Care 2001;24(7):1226-32.

40. Friedrichsen BN, Neuberger N, Lee YC, et al. Stimulation of pancreatic $\beta$-cell replication by incretins involves transcriptional induction of cyclin D1 via multiple signalingpathways. Journal of Endocrinology 2006;188(3):481-92.

41. Baggio LL, Drucker DJ. Biology of incretins: GLP-1 and GIP. Gastroenterology 2007;132(6):2131-57.
42. Herbach N, Goeke B, Schneider M, et al. Overexpression of a dominant negative GIP receptor intransgenic mice results in disturbed postnatal pancreatic islet and betacell development. Regulatory Peptides 2005;125 (1-3):103-17.

43. Kim SJ, Winter K, Nian C, et al. Glucose-dependent insulinotropicpolypeptide (GIP) stimulation of pancreatic $\beta$ - cell survival is dependent upon phosphatidylinositol 3-kinase (PI3K)/protein kinase B (PKB) signaling, inactivation of the forkhead transcription factor foxo1, and down-regulation of bax expression.Journal of Biological Chemistry 2005;280(23):22297-307.

44. Lyssenko V, Eliasson L, Kotova O, et al. Pleiotropic effects of GIP on islet function involve osteopontin. Diabetes 2011;60(9):2424-33.

45. Widenmaier SB, Kim SJ, Yang SK, et al. A GIP receptor agonist exhibits $\beta$-cell anti-apoptotic actions in rat models of diabetes resulting in improved $\beta$-cell function and glycemic control. PLoS One 2010;5(3):e9590.

46. Retnakaran R, Kramer CK, Choi H, et al. Liraglutide and the preservation of pancreatic b-cell function in early type 2 diabetes: the libra trial. Diabetes Care 2014; 37(12): 3270-8. 